\title{
The aqueous extract of Brucea javanica suppresses cell growth and alleviates tumorigenesis of human lung cancer cells by targeting mutated epidermal growth factor receptor
}

\author{
This article was published in the following Dove Press journal: \\ Drug Design, Development and Therapy \\ 3 November 2016 \\ Number of times this article has been viewed
}

\author{
Seung-Hun Kim ${ }^{1, *}$ \\ Chun-Yen Liu',* \\ Po-Wei Fan' \\ Chang-Heng Hsieh' \\ Hsuan-Yuan Lin' \\ Ming-Chung Lee ${ }^{2}$ \\ Kang Fang' \\ 'Department of Life Science, National \\ Taiwan Normal University, Taipei, \\ ${ }^{2}$ Brion Research Institute of Taiwan, \\ New Taipei City, Taiwan \\ *These authors contributed equally \\ to this work
}

\begin{abstract}
As a practical and safe herbal medicine, the seeds of Brucea javanica (L.) Merr., were used to cure patients suffering from infectious diseases such as malaria. Recent advances revealed that the herb could also be a useful cancer therapy agent. The study demonstrated that aqueous $B$. javanica (BJ) extract attenuated the growth of human non-small-lung cancer cells bearing mutant L858R/T790M epidermal growth factor receptor (EGFR). The reduced cell viability in H1975 cells was attributed to apoptosis. Transfection of EGFR small hairpin RNA reverted the sensitivities. When nude mice were fed BJ extract, the growth of xenograft tumors, as established by $\mathrm{H} 1975$ cells, was suppressed. Additional histological examination and fluorescence analysis of the resected tissues proved that the induced apoptosis mitigated tumor growth. The work proved that the BJ extract exerted its effectiveness by targeting lung cancer cells carrying mutated EGFR while alleviating tumorigenesis. Aqueous BJ extract is a good candidate to overcome drug resistance in patients undergoing target therapy.
\end{abstract}

Keywords: Brucea javanica, target therapy, epidermal growth factor receptor, human lung, herbal medicine, apoptosis

\section{Introduction}

A number of medicinal herbs exhibit anticancer activities ranging from promoting cell death to reverting multidrug resistances. ${ }^{1}$ Among them, the evergreen shrub Brucea javanica (L.) Merr. (Simaroubaceae), growing in Southeast Asia and Northern Australia, exhibits diverse biological activities. The fruits serve as traditional medicine in Indonesia, Myanmar, Thailand, and China. ${ }^{2}$ Efficient without significant side effects, the medication has been used to treat patients suffering from parasite infections and malaria in East Asian countries. ${ }^{3}$ Many studies ${ }^{4-7}$ have reported that the medication serves as a potent anticancer therapeutic. The ethanol extract from the fruit has been shown to effectively repress growth of pancreatic cancer cells. ${ }^{4}$ By combining with conventional chemotherapy drugs, the emulsion of $B$. javanica oil provided an alternative nontoxic antiproliferative agent in treating patients with lung adenocarcinoma and advanced gastric cancer., ${ }^{5,6}$ In addition, the ethyl acetate extract of the seeds has been shown to heal patients with diseases related to inflammation and allergy. ${ }^{7}$ Despite numerous reports on the versatility of the fruit in treating various types of illness, the effectiveness of aqueous $B$. javanica (BJ) extract in cancer therapy is not completely understood.
Correspondence: Kang Fang Department of Life Science, National Taiwan Normal University, 88 Ting-Chou Rd, Sec 4, Taipei II6, Taiwan

Tel +88627734 6293

Fax +8862293I 2904

Email kangfang@ntnu.edu.tw 
Tyrosine kinase inhibitors (TKIs) gefitinib and erlotinib are the first-line therapeutic agents used for treating patients with non-small cell lung cancer (NSCLC) harboring mutated epidermal growth factor receptor (EGFR). The status of $E G F R$ mutation provides a predictive biomarker of the response to gefitinib treatment. ${ }^{8}$ EGFR mutation at L858R is a prerequisite for drug sensitivity. ${ }^{9}$ This specific mutation site appears in a restricted subset of NSCLC patients that includes those of East Asian ethnicity, women, and nonsmoking individuals. ${ }^{10}$ However, patients receiving tailored target therapy gradually develop secondary mutations in EGFR, which results in relapse. ${ }^{11}$ The acquired somatic mutations at amino acid at 790 of EGFR (T790M) block steric binding of gefitinib and trigger resistance. ${ }^{12,13}$ Thus, to improve treatment, new developments aimed at overcoming the resistance stemming from double mutant EGFR at L858R/T790M in NSCLC patients, are needed to complement first-line target therapy.

To address this issue, the current study aims to find out if the aqueous BJ extract regulates the proliferation and the growth of the established xenograft tumors in H1975 cells carrying double mutant EGFR. The purpose is to identify more therapeutic approach among conventional medicines to override drug resistance in the course of progressive somatic EGFR mutation during target therapy.

\section{Materials and methods}

\section{Cell culture}

Human NSCLC cells, including H1975 (two mutations in EGFR, L858R/T790M, erlotinib-insensitive), H3255 (one mutation in EGFR, L858R, erlotinib-sensitive), A549, H1299, and H460, were acquired from American Type Culture Collection (Manassas, VA, USA) and cultured in $75 \mathrm{~cm}^{2}$ tissue culture flasks. The cells were grown in Dulbecco's Modified Eagle's Medium with supplementation of $10 \%$ fetal bovine serum (Thermo Fisher Scientific, Waltham, MA, USA), $100 \mathrm{unit} / \mathrm{mL}$ penicillin and $100 \mu \mathrm{g} / \mathrm{mL}$ streptomycin, and maintained at $37^{\circ} \mathrm{C}$ in the environment of humidified incubator with $5 \% \mathrm{CO}_{2}$. The cell media was replaced every 3 or 4 days and subcultured. Cells reaching $80 \%-90 \%$ confluence were used for experiments.

\section{Chemicals and reagents}

Sun Ten Pharmaceutical (Taichung, Taiwan) provided the aqueous extracts of the whole plant following the published procedures. ${ }^{14}$ In brief, the collected materials samples were mixed with sterile water before boiling. The supernatant following centrifugation was filtered, concentrated, and adjusted to a final concentration of $1 \mathrm{~g} / \mathrm{mL}$ before storage.
The chemicals propidium iodide (PI), ribonuclease A, trypan blue, Tris-HCl, and Triton X-100 were from Sigma-Aldrich Chemical (St Louis, MO, USA); and penicillin-streptomycin, glutamine, trypsin-ethylenediaminetetraacetic acid, and Dulbecco's Modified Eagle's Medium from Thermo Fisher Scientific.

\section{Liquid chromatography/mass spectrometry analysis and instrumental conditions}

The liquid chromatography/mass spectrometry (LC/MS) method was used to identify the major markers of bioactive substances. ${ }^{15}$ The system for analysis consisted of a LC-20AD UFLC system (Shimdzu, Kyoto, Japan) linked to a LCMS8040 triple quadrupole mass spectrometer. The running condition was designed as follows: gradient elution by the mixture of mobile phases A $(0.1 \%$ formic acid and $1 \mathrm{~g} / \mathrm{L}$ solution of ammonium acetate in water $)$ and $\mathrm{B}(0.1 \%$ formic acid and $1 \mathrm{~g} / \mathrm{L}$ solution of ammonium acetate in methanol) at minutes $0-40$ with the ratio of $100 \%-70 \%$ in $\mathrm{A}$ and $0 \%-30 \%$ in $\mathrm{B}$; at minutes $40-70$ with the ratio of $70 \%-0 \%$ in $\mathrm{A}$ and $30 \%-100 \%$ in $\mathrm{B}$; at minutes $70-70.1$ with the ratio of $0 \%-100 \%$ in $A$ and $100 \%-0 \%$ in $B$; and at minutes $70.1-80$ with the ratio of $100 \%$ in $\mathrm{A}$ and $0 \%$ in $\mathrm{B}$. The flow rate was fixed at $0.4 \mathrm{~mL} / \mathrm{min}$ and column temperature kept at $40^{\circ} \mathrm{C}$. The injection volume was adjusted at $30 \mu \mathrm{L}$ and the analytical column used a Shim-pack XR-ODS II column $(2.2 \mu \mathrm{m}$, $2 \times 100 \mathrm{~mm}$, Shimadzu). Dual ion modes (electrospray ionization, [ESI] [+] and [-]) were used in MS detection and the transmission of $(\mathrm{M}+\mathrm{H})^{+}$and $(\mathrm{M}-\mathrm{H})^{-}$was set as the optimum condition. The MS detection was arranged as full scan range (400-800 amu). The interface voltages were set at $4.5 \mathrm{kV}$ for ESI (+) and $-3.5 \mathrm{kV}$ for ESI (-). With nitrogen as nebulizing and drying gas, the flow was set at 3.0 and $10 \mathrm{~L} / \mathrm{min}$, respectively. Argon as collision-induced dissociation gas was kept at $230 \mathrm{kPa}$. Desolvation lines temperature was set at $150^{\circ} \mathrm{C}$ and heat block temperature maintained at $400^{\circ} \mathrm{C}$.

\section{Cell viability assay}

Cell viabilities were determined using trypan blue exclusion assay. To estimate viability changes, $1 \times 10^{4}$ human lung carcinoma cells were added with various concentrations of aqueous $\mathrm{BJ}$ extract and incubated at $37^{\circ} \mathrm{C}$ for 12 hours, while water alone served as control group. Cells as collected by trypsin-ethylenediaminetetraacetic acid were stained with trypan blue. Cells with exclusion of the dye were counted as viable and measured with a Countess ${ }^{\mathrm{TM}}$ counter (Thermo Fisher Scientific). The viability was determined by comparing cell numbers of treated cells with those of water control as 
percentages. The inhibition concentration, $\mathrm{IC}_{50}$, defined as concentration that inhibited $50 \%$ of cell proliferation, was calculated from a calibration curve by linear regression using Microsoft Excel.

\section{Flow cytometry by PI staining}

Cells were analyzed with FACSCalibur ${ }^{\mathrm{TM}}$ flow cytometer (BD Bioscience, San Jose, CA, USA) for determination of cell cycle distribution. A total $2 \times 10^{5}$ cells were treated with various concentrations of aqueous $\mathrm{BJ}$ extract along with water for 12 hours at $37^{\circ} \mathrm{C}$. For sample preparation, both medium and the trypsinized cells were centrifuged and the supernatant removed. The collected cells were washed with phosphatebuffered saline (PBS) and mixed with 70\% alcohol and PBS mixture, and stored at $-20^{\circ} \mathrm{C}$ for 24 hours. Before analysis, the cells were labeled with $10 \mu \mathrm{g} / \mathrm{mL}$ PI (Sigma-Aldrich) that was dissolved in PBS containing $10 \mu \mathrm{g} / \mathrm{mL}$ ribonuclease A (ICN Pharmaceutical; Costa Mesa, CA, USA) for 30 minutes in darkness. Data were analyzed by FlowJo software (Tree Star, Ashland, OR, USA).

\section{Flow cytometry by double labeling with Annexin V/fluorescein isothiocyanate and $\mathrm{PI}$}

Cells seeded in 12 -well plates at $2 \times 10^{5}$ cells per well for 16 hours were incubated with various concentrations of $\mathrm{BJ}$ extract or water for 12 hours at $37^{\circ} \mathrm{C}$. The trypsinized cells were labeled with a mixture of 1:1 ratio Annexin V/fluorescein isothiocyanate (FITC) $(20 \mu \mathrm{g} / \mathrm{mL})$ and PI $(50 \mu \mathrm{g} / \mathrm{mL})$ at room temperature for 30 minutes in darkness. The flow cytometer FACSCaliburTM (BD Bioscience) was used to determine the distribution of early and late phases of apoptotic cells. Data were analyzed with FlowJo software. The application of Annexin V/FITC (An) along with PI divides four population distributions, including the viable (An negative/PI negative), the early apoptotic (An positive/PI negative), the late apoptotic (An positive/PI positive), and the necrotic (An negative/ PI positive) cells. The sums of early and late stage percentages were counted as apoptotic cell populations.

\section{Western blotting analysis of cell lysates}

Cells as plated $\left(1 \times 10^{6}\right.$ per well $)$ in serum-free media were treated with various concentrations of aqueous BJ extract for 12 hours. The collected medium was centrifuged to remove cell debris before being lysed. The protein lysate concentrations were determined using a Bio-Rad protein assay kit (Hercules, CA, USA) with bovine serum albumin as standard. The lysates as resolved by electrophoresis were transferred to nitrocellulose membrane. The blotted membrane blocked with $0.2 \%$ skim milk for 30 minutes at room temperature was incubated overnight at $4{ }^{\circ} \mathrm{C}$ with the primary antibodies of 1:2,000 dilution in 5\% skim milk (Santa Cruz Biotechnology, Santa Cruz, CA, USA). The blots were incubated with appropriate horseradish peroxidase-conjugated secondary antibodies and visualized with enhanced chemiluminescence Western blotting substrate (Pierce, Rockford, IL, USA). The signals were recorded with enhanced LAS-4000 (FUJIFILM) apparatus and the band intensities of images analyzed using ImageJ software.

\section{TUNEL assay}

The apoptotic cell death was evaluated by terminal deoxynucleotidyl transferase-mediated dUTP nick-end labeling (TUNEL) staining. Cells treated with various concentrations of aqueous BJ extract for 12 hours were permeabilized in $10 \mathrm{mM}$ citrate buffer, $\mathrm{pH}$ 6.0. After blocking the nonspecific labeling with PBS mixture containing $2 \%$ bovine serum albumin and $0.5 \%$ NP-40, cells were incubated in TUNEL reaction solution mixed with $9 \mathrm{mM}$ dUTP, $1 \mathrm{mM}$ digoxigenin-labeled dUTP (Roche, Mannheim, Germany), $2.5 \mathrm{mM}$ cobalt chloride, $100 \mathrm{mM}$ Tris $\mathrm{pH} 7.6$ and $0.3 \mathrm{U} / \mu \mathrm{L}$ terminal deoxynucleotidyl transferase for 1 hour at $37^{\circ} \mathrm{C}$ in a humidified atmosphere. The sections were washed with PBS and incubated with a 1:200 dilution of horseradish peroxidaseconjugated digoxigenin antibody (Roche). With removal of unbound antibody, cell images were taken by fluorescence or confocal microscope. All colored TUNEL images were converted to black and white by Photoshop software, before being quantitated with Multi Gauge software (version 2.1, FUJIFILM). Sections of positively stained cells containing 50 nuclei were counted. Five slides of each concentration and control sections were recorded and three independent experiments carried out.

\section{In vivo xenograft tumor study}

Four- to six-week-old athymic female nu/nu BALB/c mice (BALB/cAnN) were purchased from the National Applied Research Laboratories (Taipei, Taiwan). The animals were housed under aseptic and ventilated conditions free of pathogens with a 12-hour light-dark cycle. All experiments were performed in accordance with the Animal Care and Use Protocols, and the study was approved by the Animal Committee of National Taiwan Normal University, Taipei, Taiwan. A total $200 \mu \mathrm{L}$ of H1975 cells $\left(5 \times 10^{6}\right.$ cells $\left./ \mathrm{mL}\right)$ mixed with Matrixgel ${ }^{\mathrm{TM}}$ Basement Membrane Matrix (Corning Incorporated, Corning, NY, USA) was injected into the dorsal leg hypodermic area in 
each mouse. Once the xenograft tumors were palpable and no less than $0.1 \mathrm{~cm}^{3}$ in volume, the mice were randomly divided into two treatment groups comprising six mice each, plus a control group. The other two groups were administered orally with aqueous BJ extract at 1 and $2 \mathrm{~g} / \mathrm{kg}$ body weight, respectively, for 7 consecutive days. The control animals received equivalent volumes of water. The health and the body weights of the animals were monitored regularly. The tumor size at each time points was measured before gavage feeding. The dimensions of the xenografts (longitudinal length and transverse width) were measured using an electronic digital caliper, and the measurements converted to the xenograft volume $\left(\pi / 6 \times\right.$ width $^{2} \times$ length). Sixteen days following final feeding, the mice were sacrificed under $\mathrm{CO}_{2}$ and the collected tumor samples resected for analysis.

\section{Statistical analysis}

All data represent mean \pm standard deviation (SD) of three individual experiments. The statistical analysis using Student's $t$-test were performed using GraphPad Prism 5.00 for Windows (GraphPad, San Diego, CA, USA). Statistical differences were considered significant with $P<0.05$.

\section{Results}

\section{LC/MS analysis of composition in} aqueous BJ extract

A typical LC/MS chromatographic fingerprint profile of aqueous BJ extract was obtained (Figure 1). Twelve major components were identified from the chromatogram. The wellseparated peaks with retention times of $<40$ minutes were identified as 1) Bruceoside D (PubChem CID: 10484578),

2) Bruceine E (PubChem CID: 122785), 3) Bruceine F
(17.5 minutes), 4) Bruceine D (PubChem CID: 441788), 5) Bruceine B (PubChem CID: 161496), and 6) Bruceine I (PubChem CID: 196839), respectively. The distinct peaks above 40 minutes included 7) Bruceine J (PubChem CID: 23656476), 8) Yadanzioside F (PubChem CID: 3000798), 9) Bruceantinol B (PubChem CID: 23656477), 10) Brusatol (PubChem CID: 73432), 11) Bruceine A (PubChem CID: 160006), and 12) Bruceoside E (PubChem CID: 3000803).

\section{Low concentrations of aqueous $B$ J extract inhibited growth of NSCLC cells bearing double mutant EGFR}

H1975 cell line is an ideal model system for studying L858R/ T790M EGFR-related TKI resistance in human NSCLC cells. Cell viabilities in different human lung cancer cells were determined using aqueous BJ extract from 1 to $5 \mathrm{mg} / \mathrm{mL}$. Among the cell lines studied, H1975 cells were sensitive to BJ treatment after 12 hours and showed concentrationdependent growth inhibition. The acquired $\mathrm{IC}_{50}$ value of $2 \mathrm{mg} / \mathrm{mL}$ of BJ in H1975 cells (Figure 2) was not detected in erlotinib-sensitive $\mathrm{H} 3255$ cells that carry EGFR of mutated L858R. Cells of wild-type EGFR were unaffected by aqueous BJ extract. The results suggested that the effectiveness of BJ extract is specific in NSCLC cells harboring L858R/ T790M EGFR.

\section{BJ extract induced sub-G, cell distribution, Annexin V FITC-positive populations and apoptotic characteristics in HI975 cells}

The palpable cytotoxicity of H1975 cells was reached as the extract concentration was increased to $2 \mathrm{mg} / \mathrm{mL}$. Thus,

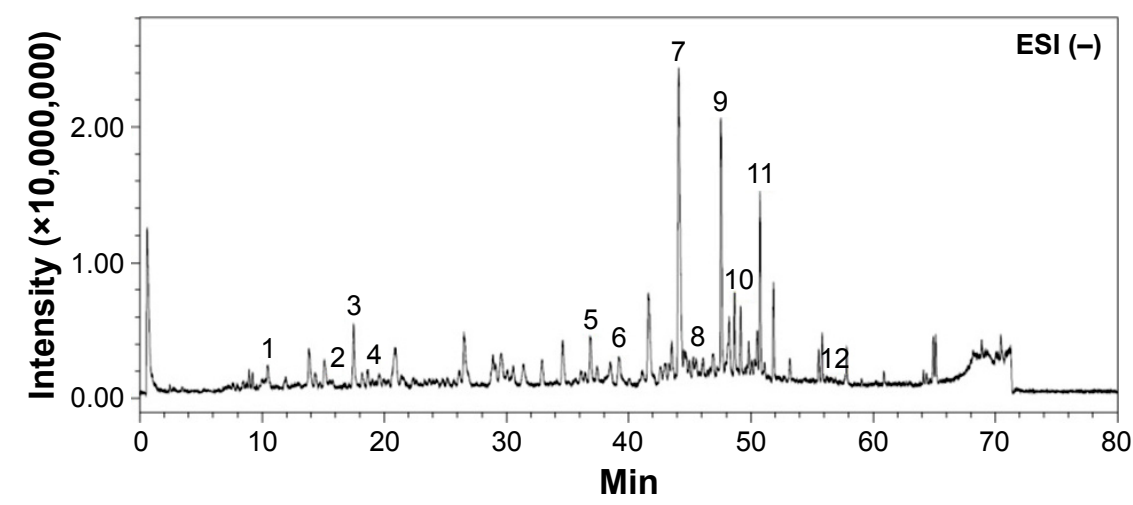

Figure I Chromatographic fingerprint analysis of the aqueous BJ extract. Twelve major components in BJ extract were recognized by LC/MS chromatogram. The peak ESI (-) mode as identified including: I) Bruceoside D (I0.6 min); 2) Bruceine E (I5.7 min); 3) Bruceine F (I7.5 min); 4 ) Bruceine D (I8.6 min); 5) Bruceine B (37.0 min); 6) Bruceine I (39.2 min); 7) Bruceine J (44.2 min); 8) Yadanzioside F (44.7 min); 9) Bruceantinol B (47.7 min); I0) Brusatol (49.2 min); II) Bruceine A (50.8 min), and I2) Bruceoside E (57.2 min).

Abbreviations: BJ, Brucea javanica; ESI, electrospray ionization; LC/MS, liquid chromatography/mass spectrometry; min, minutes. 


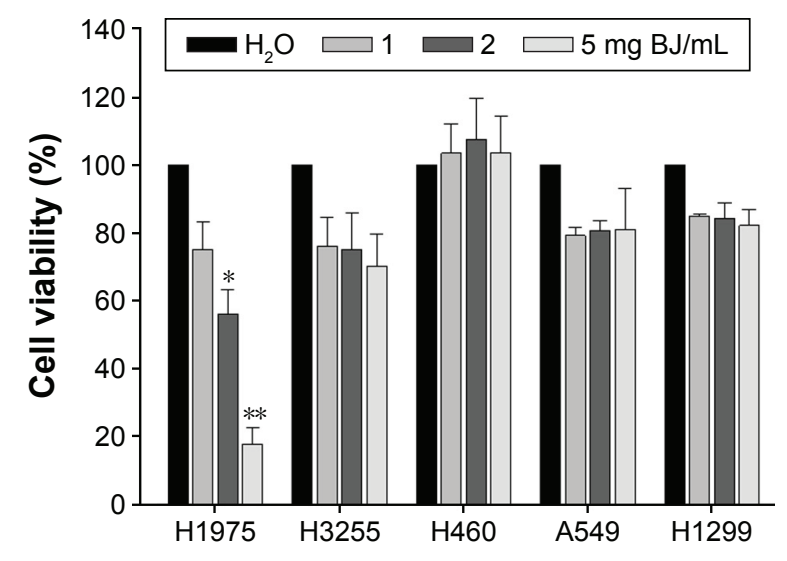

Figure 2 Dose-response growth curves of BJ extract in NSCLC HI975 cells. The increased concentration of aqueous $\mathrm{BJ}$ extracts after 12 hours of treatments reduced viable HI975 cells. The trypsinized cells were counted for the numbers of viable cells using trypan blue exclusion assay. The percentages of viabilities were obtained by comparing cell numbers at each concentration with water control that was assigned as $100 \%$. The data are the average of quadruplicate for each experiment. The results are presented as mean $\pm S D$ of three independent experiments. $* P<0.05$ and $* * P<0.01$. Abbreviations: BJ, Brucea javanica; NSCLC, non-small-cell lung cancer; SD, standard deviation.

concentrations of 1,2 , and $5 \mathrm{mg} / \mathrm{mL}$ of BJ extract were used in the subsequent experiments. Flow cytometry analysis of PI-stained cells revealed that the induced sub- $\mathrm{G}_{1}$ cell population in $\mathrm{H} 1975$ cells was dose-dependent (Figure 3A and B). Furthermore, analysis of Annexin V-FITC/PI doubly labeled $\mathrm{H} 1975$ cells showed that, as the extract concentration reached $5 \mathrm{mg} / \mathrm{mL}$ following 12 hours treatment, populations of early and late apoptotic phase were increased to $11 \%$ and $15 \%$, respectively (Figure 3C and D). The data implied that the induced apoptotic cell death contributed to the reduced viable $\mathrm{H} 1975$ cells when exposed to aqueous BJ extract.

Protein lysates of H1975 cells were subjected to Western blot analysis. BJ extract attenuated EGFR and decreased phosphorylation of both $\mathrm{EGFR}^{\mathrm{Y} 1068}$ and $\mathrm{Akt}^{\mathrm{S} 473}$ as shown in Western blot analysis (Figure 4A). The ameliorated EGFR and the reduced $\mathrm{pEGFR}^{\mathrm{Y} 1068}$ were significant with increasing BJ concentrations in comparison with water control (Figure 4B). Cleavage of poly(adenosine diphosphate ribose) polymerase (PARP) and procaspase- 3 together with formation of active caspase- 3 fragment implied apoptotic feature development. The results demonstrated that the accelerated apoptotic cell death in H1975 cells was at the expense of mutant EGFR having L858R/T790M.

\section{Knocking down EGFR impaired BJ-induced apoptotic cell death in HI975 cells}

To understand how apoptotic death was started, H1975 cells were transfected with small hairpin RNA (shRNA) targeting exon 4 of EGFR (RNA sequence: GAGAAU GUGGAAUACCUAAGG) along with nonspecific shRNA as control (RNA sequence: CCGGACACUCGAGCAC UUUUUG). The recovered viable cells transfected with EGFR shRNA were more than cells with nonspecific shRNA following BJ treatment (Figure 5A). Aqueous BJ extract accentuated diminution of phosphorylated EGFR $^{\mathrm{Y} 1068}$ in H1975 cells following EGFR shRNA transfection (Figure 5B). The reduced PARP cleavage signified that knocking down EGFR incapacitated BJ sensitivity. The results implied that BJ targeted intrinsic EGFR with double mutation and orchestrated cell death.

\section{Orally administrated BJ reduced xenograft tumor growth of HI975 cells in nude mice}

The sizes of xenograft tumors of H1975 cells were diminished when feeding mice with BJ extracts. The continuous tumor development was repressed in animals when fed with $2 \mathrm{~g} \mathrm{BJ} / \mathrm{kg}$ compared with those with water (Figure 6A). No comparable body weight loss in nude mice orally administered with aqueous BJ extract was seen relative to those administered water treatment during experiment durations (Figure 6B). The increased BJ dosage proportionately reduced weights of the dissected tumors (Figure 6C).

\section{The increased apoptosis and the regressed EGFR in xenograft tumors}

As shown in hematoxylin-eosin staining of the histological sections, mice administered with aqueous BJ extract induced apoptotic cell death in xenograft tumors. The microscopic images of tumor sections in mice fed with water showed packed viable cells, whereas those with $2 \mathrm{~g} \mathrm{BJ} / \mathrm{kg}$ exhibited increasingly apoptotic characteristics, including cytoplasm condensation, pyknotic nuclei, and interstitial space enlargement. Some apparent engulfed apoptotic bodies were visible (Figure 7A). Cytochrome $c$ release from mitochondria were visualized by confocal microscopy analysis. In tumor section images, the induced fluorescent puncta with coalesced green mitochondria marker mitotracker and the released red cytochrome $c$ suggested progressive apoptotic cell death of $\mathrm{H} 1975$ tumors in mice given increasing BJ extract (Figure 7B). The increasingly administered BJ depleted fluorescent EGFR (Figure 7C) and pEGFR $^{\mathrm{Y} 1068}$ (Figure 7D) as evidenced by antibody detection of tumor dissections.

The enriched TUNEL fluorescence in the resected specimen implied induced apoptotic death in H1975 xenograft 
A

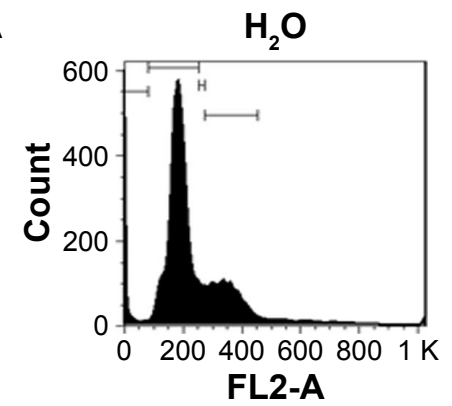

2

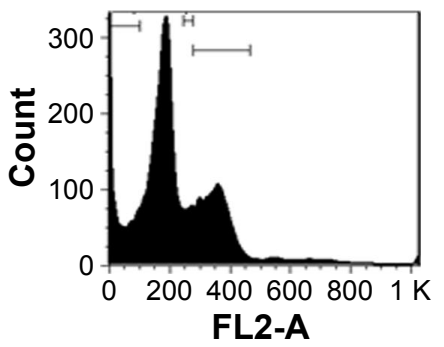

C

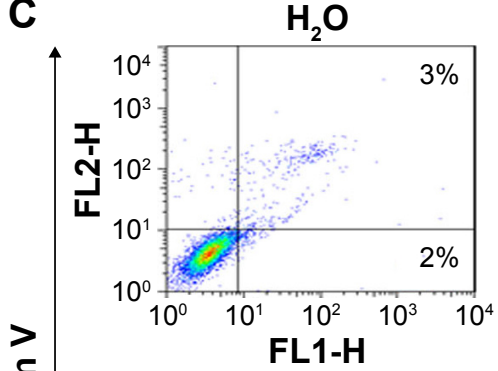

$2 \mathrm{mg} / \mathrm{mL}$

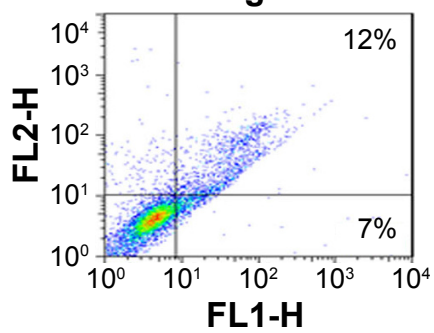

1

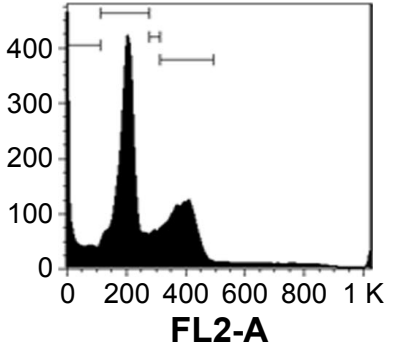

$5 \mathrm{mg} / \mathrm{mL}$

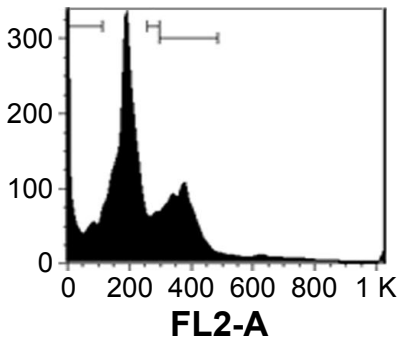

$1 \mathrm{mg} / \mathrm{mL}$

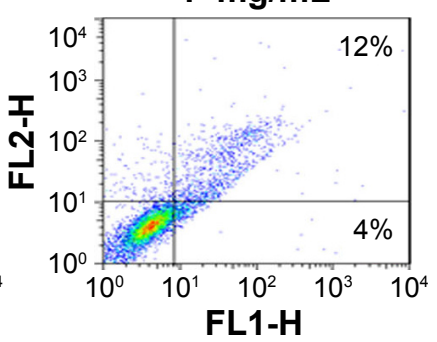

FL1-H

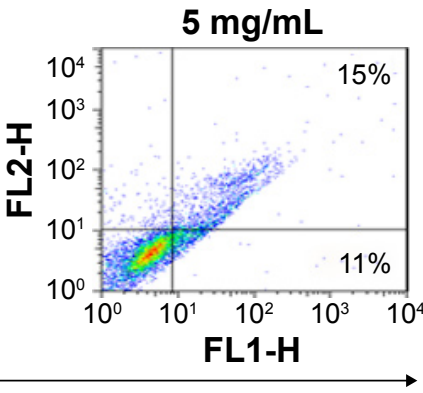

B
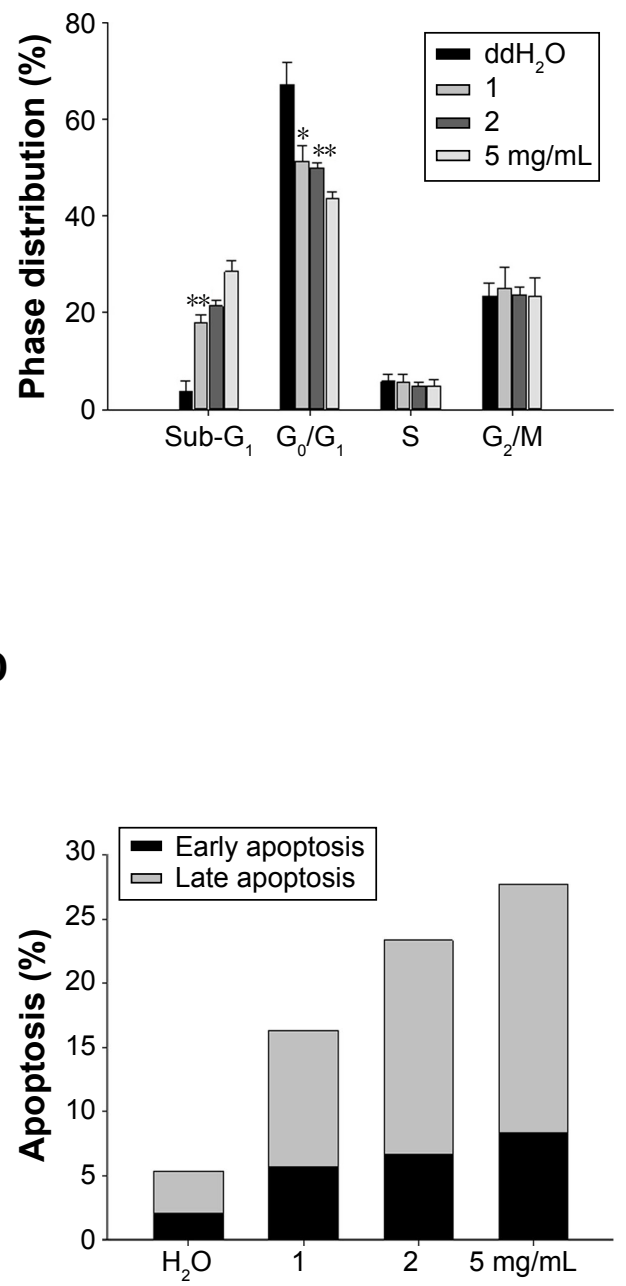

PI

Figure 3 The aqueous BJ extract increased apoptotic distributions of HI 975 cells by (A) dose-dependent appearance of sub-G, cells. Human NSCLC cells HI 975 cultured in I\% serum-supplemented DMEM were incubated with various concentrations of BJ extract (I, 2, and $5 \mathrm{mg} / \mathrm{mL}$ ) or water for 12 hours before being labeled with PI followed by flow cytometry analysis. (B) Cell cycle distribution. The percentages of cell cycle distribution in HI 975 cells following treatment were analyzed. The results of BJ treatment were compared with those of water control. $* P<0.05$. $* * P<0.0$ I. (C) Histograms of two-dimensional flow cytometry $\mathrm{HI} 975$ cells were treated with various concentrations of BJ for 12 hours and the trypsinized cells labeled with Annexin V and PI were analyzed by flow cytometry. (D) Quantitative analysis of the apoptotic cell populations. Populations of early (dark) and late (light) apoptotic distributions in HI975 cells treated with various concentrations of BJ were analyzed. The results were expressed as mean values from three independent experiments.

Abbreviations: BJ, Brucea javanica; DMEM, Dulbecco's Modified Eagle's Medium; NSCLC, non-small cell lung cancer; PI, propidium iodide.

tumors in mice administered with BJ extract (Figure 8A). The increased fluorescence intensities meant enhanced apoptotic index relative to groups with water (Figure 8B). On the other hand, $\mathrm{BJ}$ extract inhibited tumor proliferation as suggested by the reduced mitotic index proliferating cell nuclear antigen (PCNA) (Figure 8C). The decreased nucleus PCNA staining as shown suggested that tumor growth alleviation is attributed to apoptosis (Figure 8D).

\section{Discussion}

The indigenous ethnic medicines have received wide attention and been identified with new anticancer prospect. Recent efforts have advanced herbal medicine research to complement cancer therapy treatment. An important objective of drug development is to uncover more potent regimens that overcome the arising resistance during target therapy. ${ }^{16}$ The emulsified formula of $\mathrm{BJ}$ has been approved to treat various 

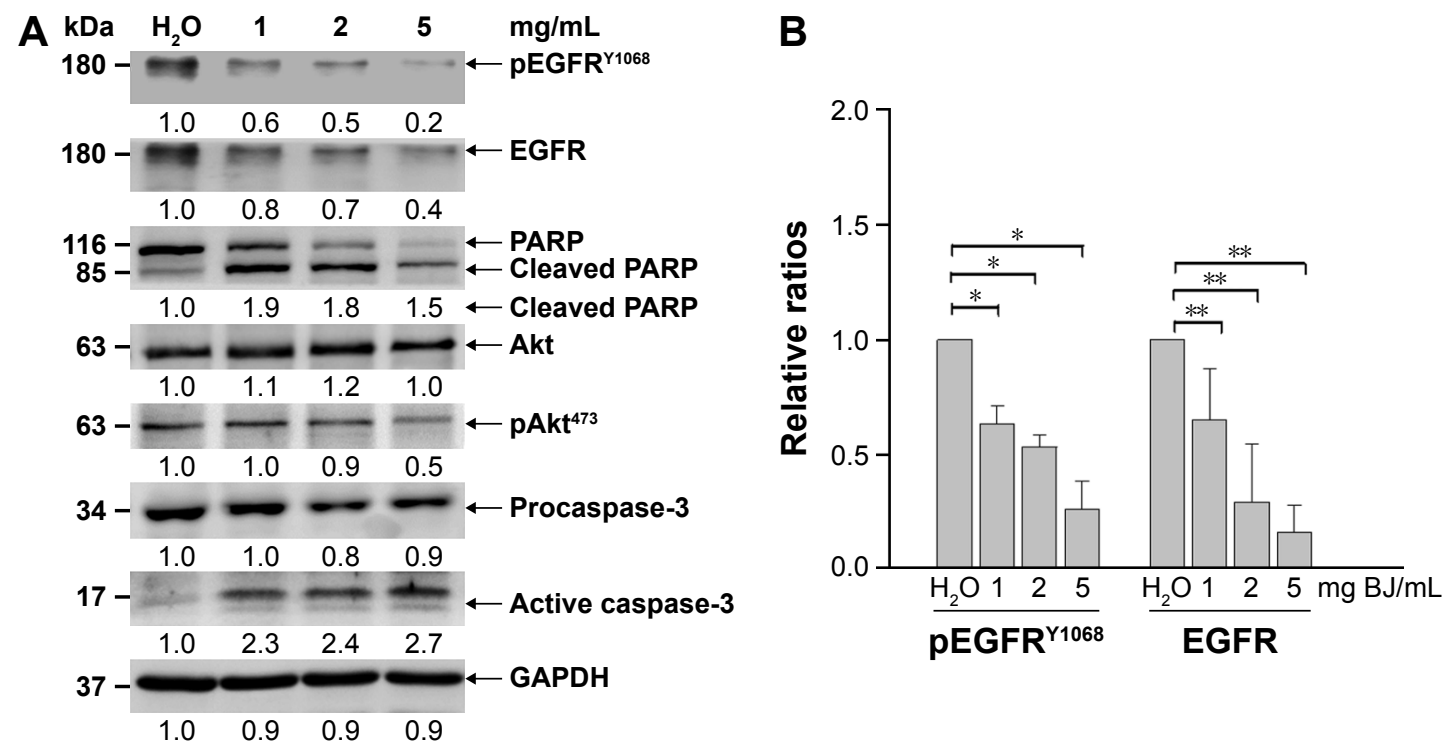

Figure $4 \mathrm{BJ}$ induced apoptosis and reduced EGFR and PEGFR ${ }^{\text {Y } 1068}$ levels in HI975 cells. (A) Western blot analysis. The protein lysates from HI975 cells as treated with $\mathrm{I}, 2$, and $5 \mathrm{mg} / \mathrm{mL}$ of BJ extract for 12 hours were collected and used for Western blot analysis. The blots were incubated with various primary antibodies, including EGFR, phosphorylated EGFR ${ }^{Y 1068}$, Akt, phosphorylated $\mathrm{Akt}^{\mathrm{S} 473}$, caspase-3, and PARP as specified, which were followed by HRP-conjugated secondary antibodies. GAPDH was used as loading control. The blots were visualized by ECL detection system. (B) Densitometric determination of EGFR and phosphorylated EGFR ${ }^{\text {Y1068 }}$ amelioration. The densitometric ratios of EGFR and phosphorylated EGFR ${ }^{Y 1068}$ in $\mathrm{HI} 975$ cells from Western blot analysis were obtained by first normalizing individual band intensity at each concentration to that of the loading control and compared with those of water treatment. The results were expressed as mean values of three independent experiments $(* P<0.05$, $* * P<0.0$ I, unpaired Student's t-test as compared with control water).

Abbreviations: BJ, Brucea javanica; EGFR, epidermal growth factor receptor; GAPDH, glyceraldehyde 3-phosphate dehydrogenase; PARP, poly(adenosine diphosphate ribose) polymerase; pEGFR, phosphorylated EGFR.

types of cancer, such as lung cancer diagnosed at middle or late stage. ${ }^{17,18}$ To extend the scope, the study reported that the aqueous BJ extract inhibited the growth of human lung cancers with somatic mutation of EGFR. The findings offered new dimension for the existing herbal medicine to override the evolving resistance during target therapy.
Being a transmembrane protein endowed with kinase activity on downstream effectors, EGFR binds to ligand that leads to receptor dimerization and phosphorylation of tyrosine residues at the cytosolic domains. The phosphorylated tyrosine residues function as a kinase that activates downstream intracellular signals, such as extracellular-regulated kinase.
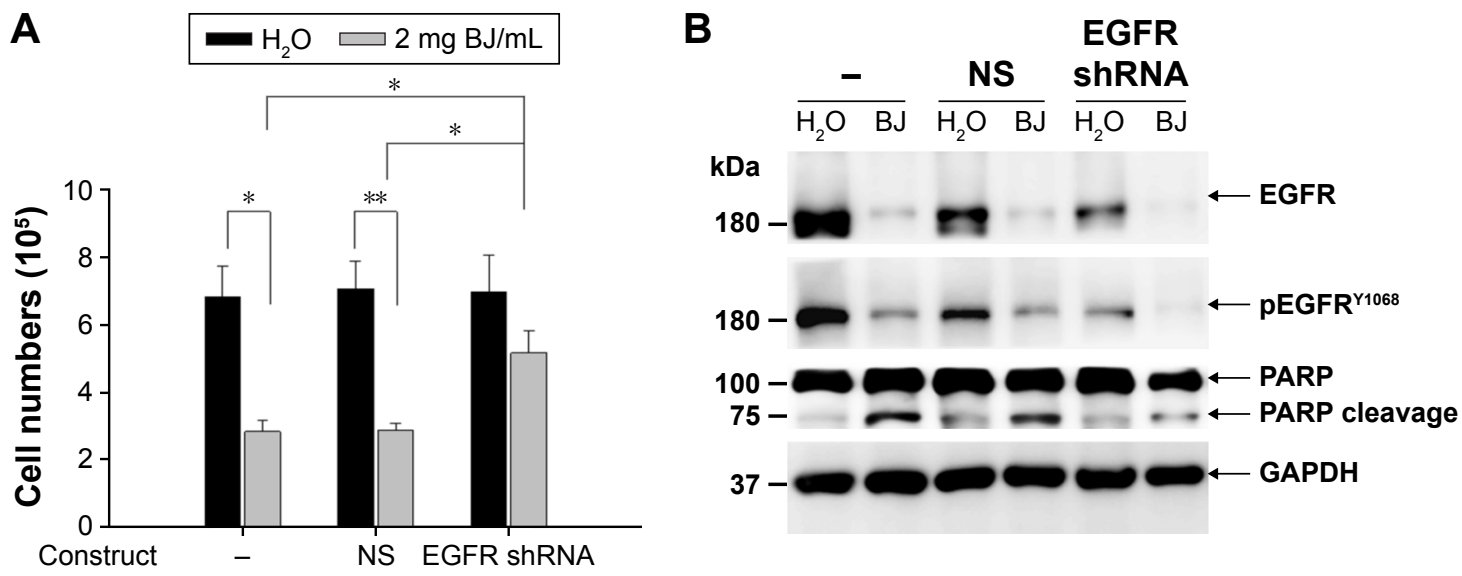

Figure 5 EGFR shRNA reduced BJ sensitivities by suppressing apoptosis. (A) Viability determination. HI 975 cells transfected with EGFR shRNA or NS control for 24 hours were treated with $2 \mathrm{mg} / \mathrm{mL}$ of BJ extract for 12 hours and the collected cells counted by trypan blue exclusion assay. Symbol (-) meant no transfection. The results were expressed as mean values from three independent experiments. $* P<0.05$ and $* * P<0.01$ indicated significant difference between groups. (B) Western blot analysis. Proteins of HI 975 cells transfected with EGFR shRNA and NS control before being treated with BJ extract $(2 \mathrm{mg} / \mathrm{mL})$ or water were subjected to Western blot analysis. The antibodies included EGFR, phosphorylated EGFR ${ }^{\text {Y } 1068}$, PARP and loading control GAPDH. Symbol (-) indicated no transfection.

Abbreviations: BJ, Brucea javanica; EGFR, epidermal growth factor receptor; GAPDH, glyceraldehyde 3-phosphate dehydrogenase; PARP, poly(adenosine diphosphate ribose) polymerase; NS, non-specific; shRNA, small hairpin RNA. 


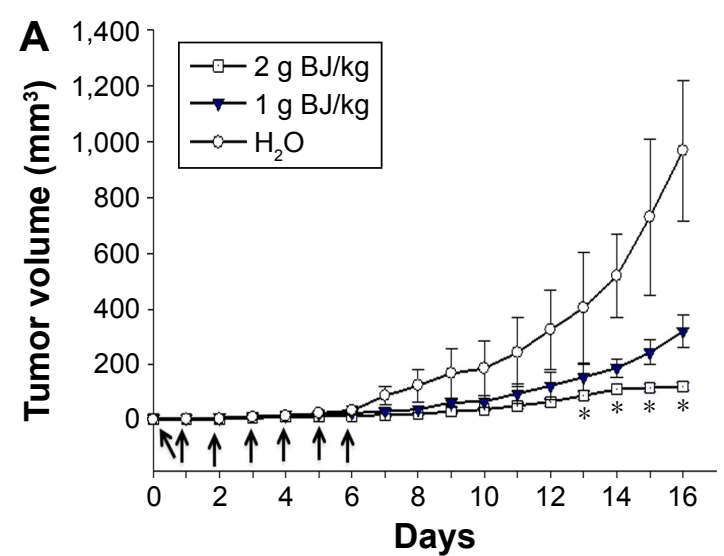

B
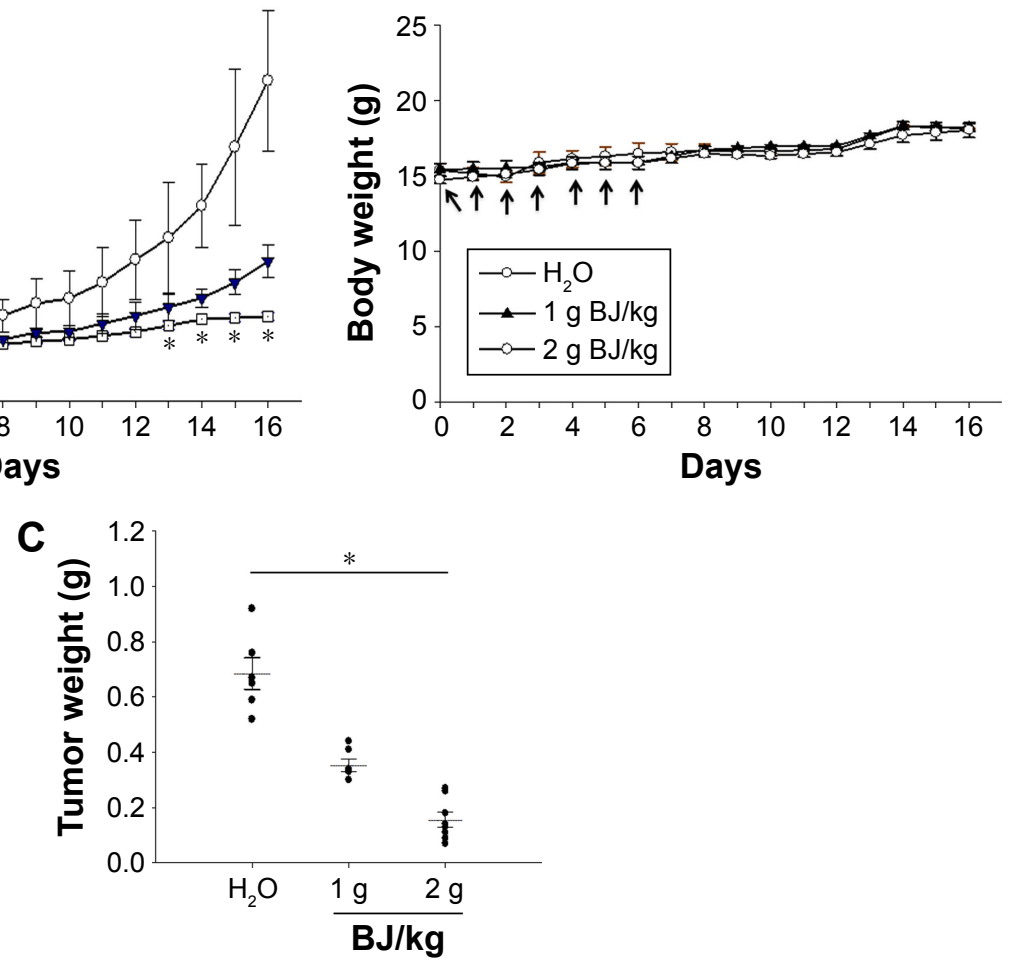

Figure 6 Oral administration of BJ reduced xenograft tumor growth. (A) BJ extract suppressed the growth of xenograft tumors in animal models. Nude BALB/c mice with established xenograft tumors of HI975 cells were administered orally with I and $2 \mathrm{~g} \mathrm{BJ} / \mathrm{kg}$ daily for 7 consecutive days (arrows). The graph represented tumor growth variation ( $y$-axis) from the start of feeding ( $x$-axis). Mice fed with aqueous BJ extract showed reduced tumor growth compared with those with water. $* P<0.05$ indicated significant difference in the measured tumor volumes between mice fed with $2 \mathrm{~g} \mathrm{BJ} / \mathrm{kg}$ and those with water from three individual experiments with six mice in each group. (B) The body weights of the nude mice. No significant difference was seen in the average body weight of mice with established xenograft $\mathrm{HI} 975$ tumors orally administered water, $\mathrm{I}$ and $2 \mathrm{~g} \mathrm{BJ} / \mathrm{kg}$ daily for 7 consecutive days (arrow). The graph represented variations of mice weight ( $y$-axis) from the start of feeding in days ( $x$-axis). (C) The decreased resected tumor weights. Tumor weights of the resected xenograft tumors were suppressed in mice with gavage feeding of $B J$ compared with those of water. The horizontal bars represented mean values of tumor mass as collected following different treatments. $* P<0.05$ indicated significant weight difference between mice fed with $2 \mathrm{~g} B \mathrm{~J} / \mathrm{kg}$ and those with water. Each graph is representative of three independent experiments.

Abbreviation: BJ, Brucea javanica.

Mutated EGFR frequently occurred in lung adenocarcinoma patients, especially those of Asian ethnicity, females, and nonsmokers. Both gefitinib and erlotinib serve as the first-line TKI therapy to treat advanced NSCLC patients harboring EGFR mutation by preventing dimerization and phosphorylation of the receptor. ${ }^{18}$ As first choice for target therapy, gefitinib and erlotinib bind to L858R EGFR of lung cancer cells, thereby making treatment successful. ${ }^{18,19}$ Treatment of TKI significantly prolonged survival rates of lung cancers patients with the specifically mutated EGFR. ${ }^{20}$ Despite their distinct response, lung cancer patients receiving target therapy eventually develop resistance due to the evolved secondary T790M mutation. The rate arises at a median of $10-13$ months that occurs in more than $50 \%$ of the patients. ${ }^{21}$ In order to overcome the high concentration toxicity and resistance because of the accumulated somatic mutation of T790M EGFR, new development of safer and more useful medication following target therapy is imperative. The study showed that BJ suppressed cell viabilities only in H1975 cells.
The diminished EGFR intensities and the repressed EGFR ${ }^{\text {Y } 1068}$ phosphorylation account for the growth rate reduction.

BJ increased sub- $\mathrm{G}_{1}$ cells and Annexin V-positive populations in H1975 cells. More work showed that the aqueous extracts attenuated Akt signaling and induced procaspase-3 cleavage. Active caspase- 3 is required to elicit and execute apoptosis by cleaving cellular proteins at appointed aspartate residues. ${ }^{22}$ Western blot analysis showed that both PARP and procaspase- 3 were cleaved following BJ treatment that triggered subsequent apoptosis. The appearance of apoptotic caspase-3 fragment corroborated the decreased viable cells. Knocking-down EGFR in H1975 cells inhibited PARP cleavage, reduced subsequent apoptotic cell death and suppressed BJ sensitivity. The results supported the vital role of the mutant EGFR in H1975 cells during BJ-induced apoptosis.

More experiments showed that orally administered aqueous BJ extract inhibited the growth of the established xenograft H1975 tumors without affecting the healthiness of mice. Aqueous BJ administration diminished EGFR intensities 
A
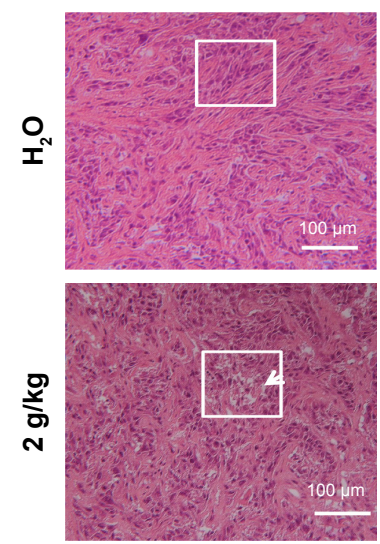

C
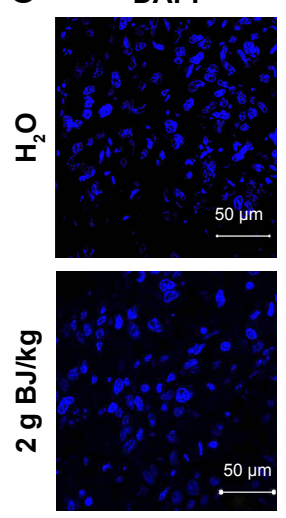

Zoom-in
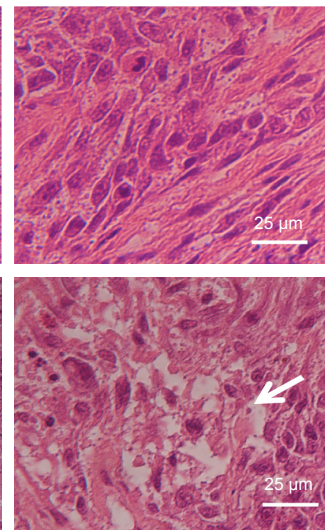

EGFR
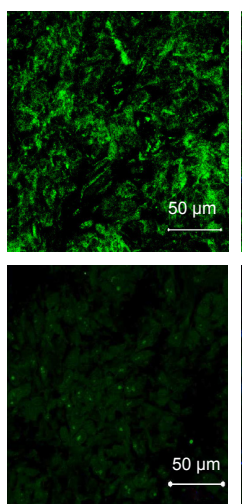

Merge
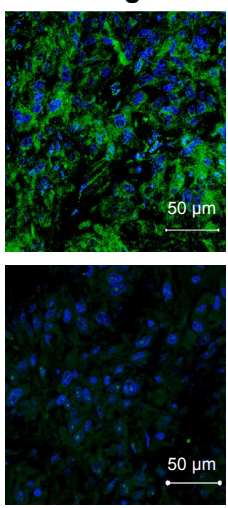

B
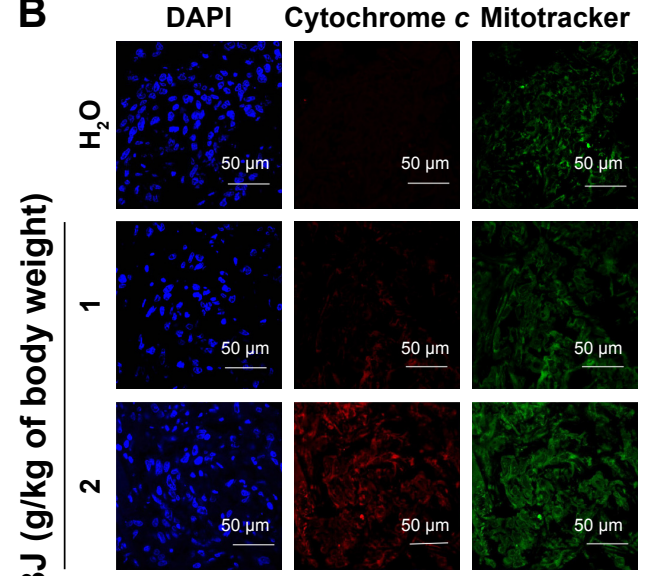

$50 \mu \mathrm{m}$
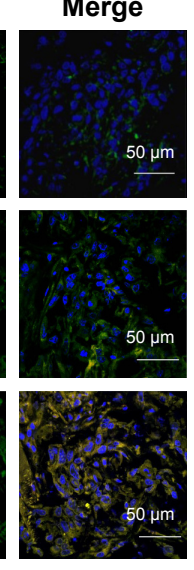

D DAPI

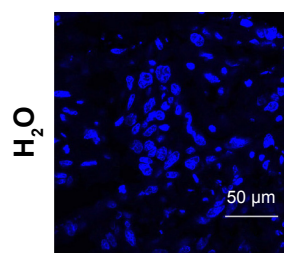

pEGFR $^{\mathrm{Y} 1068}$
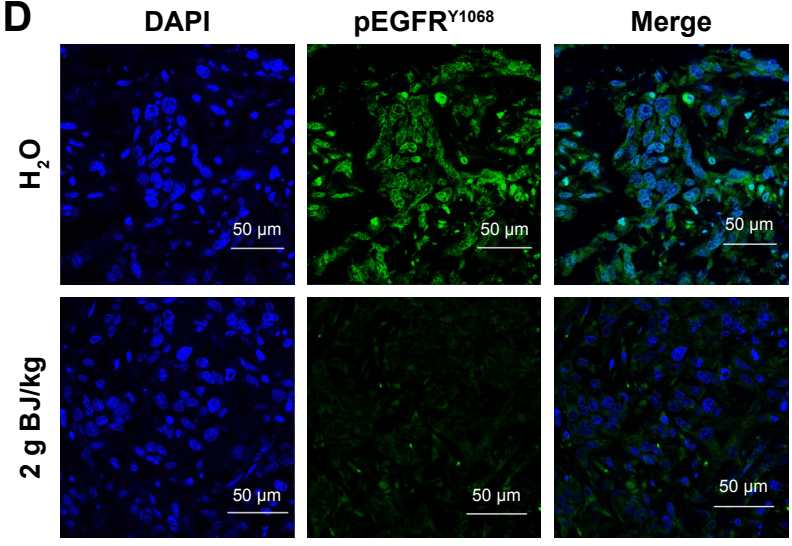

Figure 7 Histological and fluorescence examination of the reduced tumors. (A) Examination by HE staining. The paraffin-embedded $\mathrm{H} / 975$ tumors treated with $\mathrm{BJ}(2 \mathrm{~g} / \mathrm{kg}$ ) and water control were dissected, stained by HE and analyzed by confocal microscopy (scale bar $=100 \mu \mathrm{m}$ ). The rectangular inset of the image were zoomed-in to the right of the panel for each treatment (scale bar $=25 \mu \mathrm{m}$ ). The white arrow signifies apoptotic body location. (B) Release of mitochondrial cytochrome $c$ in tumors treated with BJ. The dissected specimens of HI975 tumors as treated with BJ (I and $2 \mathrm{~g} / \mathrm{kg}$ ) and water control were fixed and incubated with antibody against cytochrome c followed by staining with secondary antibody conjugated with TRITC (red). The slides were counter-stained with mitotracker (green) and DAPI (blue) before being analyzed by confocal microscopy. The merged images of red color cytochrome $c$ and green color mitochondria signified the appearance of puncta (yellow), while blue color indicates nucleus (scale bar $=50 \mu \mathrm{m}$ ). (C) Images of immunofluorescent EGFR HI 975 tumor sections from mice fed with $2 \mathrm{~g} / \mathrm{kg}$ of BJ and water control were incubated with EGFR antibody (green) followed by FITC-conjugated secondary antibody treatment before being counterstained with DAPI (blue) (scale bar =50 $\mu \mathrm{m}$ ). (D) Images of immunofluorescent pEGFR ${ }^{\text {YIO68 }}$ HI975 tumor sections from mice fed with $2 \mathrm{~g} / \mathrm{kg}$ of BJ and water control were incubated with pEGFR ${ }^{\text {Ylo68 }}$ antibody (green) followed by FITC-conjugated secondary antibody incubation before being counterstained with DAPI (blue) (scale bar $=50 \mu \mathrm{m}$ ).

Abbreviations: BJ, Brucea javanica; DAPI, 4',6-diamidino-2-phenylindole; EGFR, epidermal growth factor receptor; FITC, fluorescein; HE, hematoxylin and eosin; TRITC, tetramethylrhodamine.

and depressed phosphorylation levels in the collected tumor resections. The increment of cytochrome $c$ and TUNEL staining plus the declined PCNA marker as shown in confocal images further validated that BJ eliminated H1975 tumor growth by apoptotic cell death. Overall, the aqueous extract of BJ effectively overcome drug resistance by targeting $E G F R$ with secondary mutation during therapy. The results suggested that aqueous BJ extract rich in various forms of quassinoids prompted apoptosis in H1975 cells with L858R/T790M EGFR. One of the major components in the aqueous BJ extract, brusatol, is capable of overcoming drug resistance by decreasing nuclear factor erythroid 2 p45-related factor 2, thereby enhancing the cytotoxic effect of numerous chemotherapeutic agents. ${ }^{23} \mathrm{~A}$ report showed that cationic nanoemulsions of $\mathrm{BJ}$ oil mixture can be an effective delivery system that enhances the oral bioavailability with promising anticancer prospect. ${ }^{24}$ A recent study has proven that aqueous extract of the BJ plant suppressed the growth of liver cancer by reducing cell proliferation and activating apoptosis. ${ }^{14}$ The work described here illuminates more aspects of the plant as an effective therapeutic approach in treating lung cancer. The study further exemplified that, as an alternative medicine, BJ complements the existing cancer therapy by targeting cells with mutant EGFR.

\section{Conclusion}

The discovery underscored the potency of aqueous BJ in eliminating cancer cell growth and alleviating tumor growth. $\mathrm{BJ}$ extract targeted somatic EGFR mutation in NSCLC cells 
A
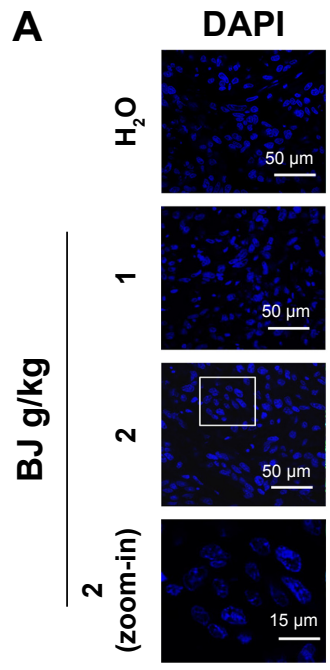

C
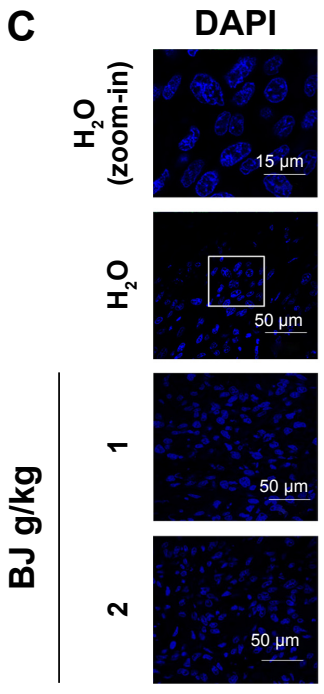
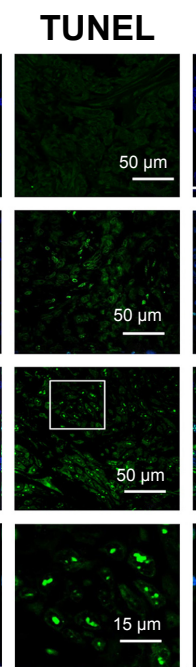

PCNA
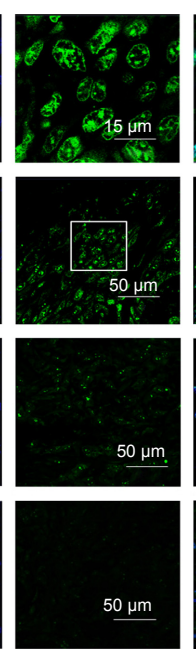
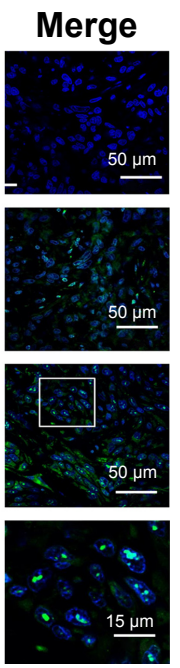

Merge
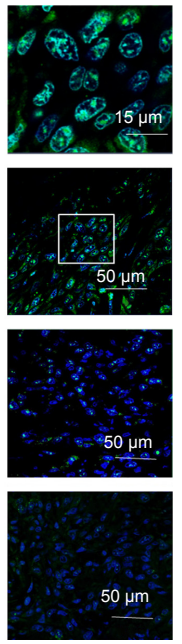

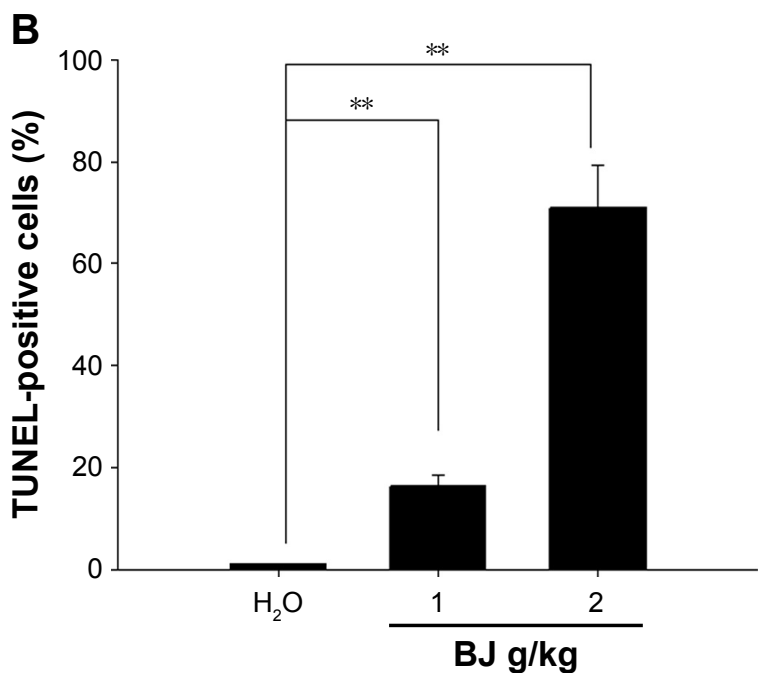

D

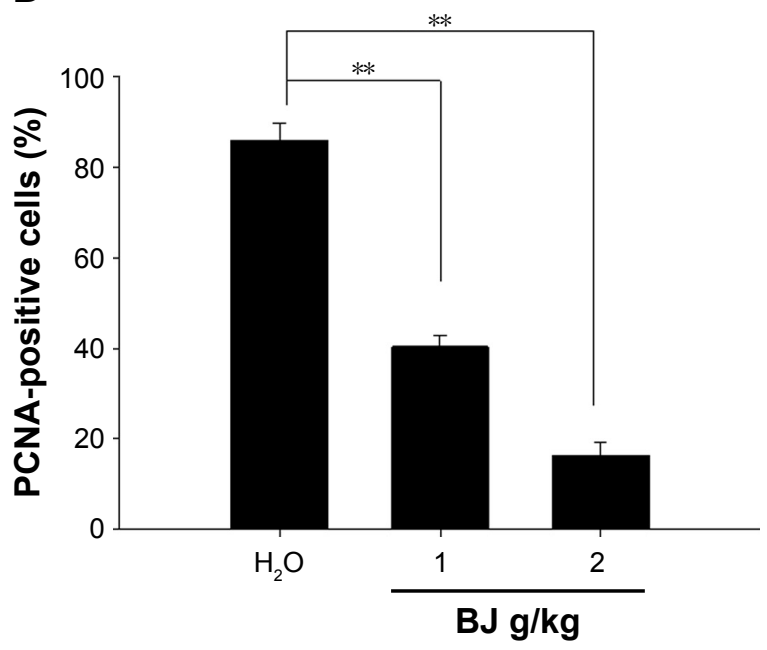

Figure 8 Tumor growth suppression by apoptosis. (A) Dose-dependent increase of TUNEL staining. The tumors removed from the mice fed with water, I and $2 \mathrm{~g} B \mathrm{~J} / \mathrm{kg}$ were frozen, resected, fixed, and subjected to TUNEL experiment for confocal microscopy analysis. The apoptotic bodies (green) were counterstained with DAPI (blue) and visualized (scale bar $=50 \mu \mathrm{m}$ ). The image of rectangular inset of tumor sections in mice treated with $2 \mathrm{~g} \mathrm{BJ} / \mathrm{kg}$ was zoomed-in to the bottom of the panel (scale bar $=15 \mu \mathrm{m}$ ). (B) BJ increased TUNEL-positive nuclei intensities. The number of fluorescent TUNEL-positive cells counted in each field was I00, as marked by DAPI staining. The numbers of TUNEL-positive cells at various BJ concentrations were the average of at least three different fields. The data were expressed as the mean of three individual experiments $(* * P<0.0 I)$. (C) Dose-dependent decrease of stained nucleus PCNA. Tumor sections were incubated with rabbit antibody against PCNA followed by FITC-conjugated secondary antibody. The slides with PCNA fluorescence (green) were counterstained with DAPI (blue) before being analyzed by confocal microscopy (scale bar $=50 \mu \mathrm{m}$ ). The images of rectangular inset of tumor sections in mice treated with water were zoomed-in to the top of the panel (scale bar =15 $\mu \mathrm{m}$ ). (D) BJ decreased mitotic index PCNA signals in nucleus. The number of fluorescent nucleus PCNA-positive cells counted in each field was I00, as marked by DAPI staining. The numbers at various $B J$ concentrations were the average of at least three different fields. The data were expressed as the mean of three individual experiments $(* * P<0.0 \mathrm{I})$.

Abbreviations: BJ, Brucea javanica; DAPI, 4',6-diamidino-2-phenylindole; PCNA, proliferating cell nuclear antigen; TUNEL, transferase-mediated dUTP nick-end labeling.

by inducing apoptosis and suppressing tumorigenesis. The study highlighted the value and potential application of BJ as an alternative to treat human lung cancer refractive to target therapy. The findings promise more innovative prospect of the conventionally available herbal medicine to improve relapse and prolong survival rates of cancer patients.

\section{Acknowledgments}

The work is supported by grants from Ministry of Science and Technology, Executive Yuan, ROC (MOST
104-2320-B-003-001) and National Taiwan Normal University (102T3040B2, 103T3040D2 and 104T3040C2). Technical assistance of inverted and confocal laser microscopy from the College of Life Science Instrumentation Center, National Taiwan Normal University was appreciated. The assistance of the National RNAi Core Facility (Academia Sinica, Taipei, Taiwan) is acknowledged.

\section{Disclosure}

The authors report no conflicts of interest in this work. 


\section{References}

1. Han SY, Zhao MB, Zhuang GB, Li PP. Marsdenia tenacissima extract restored gefitinib sensitivity in resistant non-small cell lung cancer cells. Lung Cancer. 2012;75(1):30-37.

2. Gao H, Lamusta J, Zhang WF, et al. Tumor cell selective cytotoxicity and apoptosis induction by an herbal preparation from Brucea javanica. North Am J Med Sci. 2011;4(2):62-66.

3. Zhao L, Li C, Zhang Y, Wen Q, Ren D. Phytochemical and biological activities of an anticancer plant medicine: Brucea javanica. Anticancer Agents Med Chem. 2014;14(3):440-458.

4. Lau ST, Lin ZX, Zhao M, Leung PS. Brucea javanica fruit induces cytotoxicity and apoptosis in pancreatic adenocarcinoma cell lines. Phytother Res. 2008;22(4):477-486.

5. Liu J, Huang XE, Tian GY, et al. Phase II study on safety and efficacy of Yadanzi ${ }^{\circledR}$ (Javanica oil emulsion injection) combined with chemotherapy for patients with gastric cancer. Asian Pac J Cancer Prev. 2013;14(3):2009-2012.

6. Lu YY, Huang XE, Cao J, et al. Phase II study on Javanica oil emulsion injection (Yadanzi ${ }^{\circledR}$ ) combined with chemotherapy in treating patients with advanced lung adenocarcinoma. Asian Pac J Cancer Prev. 2013;14(8):4791-4794.

7. Yang J, Li S, Xie C, et al. Anti-inflammatory activity of ethyl acetate fraction of the seeds of Brucea javanica. J Ethnopharmacol. 2013; 147(2):442-446.

8. Suda K, Mitsudomi T. Role of EGFR mutations in lung cancers: prognosis and tumor chemosensitivity. Arch Toxicol. 2015;89(8):1227-1240.

9. Rolfo C, Giovannetti E, Hong DS, et al. Novel therapeutic strategies for patients with NSCLC that do not respond to treatment with EGFR inhibitors. Cancer Treat Rev. 2014;40(8):990-1004.

10. Soucheray M, Capelletti M, Pulido I, et al. Intratumoral heterogeneity in EGFR mutant NSCLC results in divergent resistance mechanisms in response to EGFR tyrosine kinase inhibition. Cancer Res. 2015;75(20): 4372-4383.

11. Yun CH, Boggon TJ, Li Y, et al. Structures of lung cancer-derived EGFR mutants and inhibitor complexes: mechanism of activation and insights into differential inhibitor sensitivity. Cancer Cell. 2007;11(3): 217-227.

12. Liu SV, Subramaniam D, Cyriac GC, et al. Emerging protein kinase inhibitors for non-small cell lung cancer. Expert Opin Emerg Drugs. 2014;19(1):51-65.
13. Zhang W, Lei P, Dong X, Xu C. The new concepts on overcoming drug resistance in lung cancer. Drug Des Devel Ther. 2014;8:735-744.

14. Chen JH, Kim SH, Fan BW, Liu CY, Hsieh CH, Fang K. The aqueous extract of Chinese medicinal herb Brucea javanica suppresses the growth of human liver cancer and the derived stem-like cells by apoptosis. Drug Des Devel Ther. 2016;10:2003-2013.

15. Lien CY, Chuang TY, Hsu CH, et al. Oral treatment with the herbal formula B307 alleviates cardiac toxicity in doxorubicin-treated mice via suppressing oxidative stress, inflammation, and apoptosis. OncoTargets Ther. 2015;8:1193-1210.

16. Shih JY, Gow CH, Yang PC. EGFR mutation conferring primary resistance to gefitinib in non-small-cell lung cancer. $N$ Engl J Med. 2005; 353(2):207-208

17. Nie YL, Liu KX, Mao XY, Li YL, Li J, Zhang MM. Effect of injection of Brucea javanica oil emulsion plus chemoradiotherapy for lung cancer: a review of clinical evidence. J Evid Based Med. 2012;5(4):216-225.

18. Zhou C, Wu YL, Chen G, et al. Erlotinib versus chemotherapy as first-line treatment for patients with advanced EGFR mutation-positive non-small-cell lung cancer (OPTIMAL, CTONG-0802): a multicentre, open-label, randomised, phase 3 study. Lancet Oncol. 2011;12(8): $735-742$.

19. Lynch TJ, Bell DW, Sordella R, et al. Activating mutations in the epidermal growth factor receptor underlying responsiveness of non-small-cell lung cancer to gefitinib. New England J Med. 2004; 350(21):2129-2139.

20. Suda K, Mitsudomi T. Role of EGFR mutations in lung cancers: prognosis and tumor chemosensitivity. Arch Toxicol. 2015;89:1227-1240.

21. Armour AA, Watkins CL. The challenge of targeting EGFR: experience with gefitinib in nonsmall cell lung cancer. Eur Resp Rev. 2010; 19(117):186-196.

22. Ghavami S, Hashemi M, Ande SR, et al. Apoptosis and cancer: mutations within caspase genes. J Med Genet. 2009;46(8):497-510.

23. Furfaro AL, Traverso N, Domenicotti C, et al. The Nrf2/HO-1 Axis in cancer cell growth and chemoresistance. Oxid Med Cell Longev. 2016; 2016:1958174.

24. Liu TT, Mu LQ, Dai W, Wang CB, Liu XY, Xiang DX. Preparation, characterization, and evaluation of antitumor effect of Brucea javanica oil cationic nanoemulsions. Int J Nanomedicine. 2016;11:2515-2529.
Drug Design, Development and Therapy

\section{Publish your work in this journal}

Drug Design, Development and Therapy is an international, peerreviewed open-access journal that spans the spectrum of drug design and development through to clinical applications. Clinical outcomes, patient safety, and programs for the development and effective, safe, and sustained use of medicines are the features of the journal, which

\section{Dovepress}

has also been accepted for indexing on PubMed Central. The manuscript management system is completely online and includes a very quick and fair peer-review system, which is all easy to use. Visit http://www.dovepress.com/testimonials.php to read real quotes from published authors. 